\title{
Proposal for environmental mixture risk assessment in the context of the biocidal product authorization in the EU
}

Thomas Backhaus $^{1 *}$, Rolf Altenburger ${ }^{2}$, Michael Faust $^{3}$, Daniel Frein ${ }^{4}$, Tobias Frische $^{5}$, Per Johansson ${ }^{6}$, Anja Kehrer ${ }^{7}$ and Tobias Porsbring ${ }^{8}$

\begin{abstract}
Background: The EU Council and Parliament recently agreed on a new regulation that will implement a new EU-wide, harmonized system for the authorization for biocidal products. Such products are in most cases multi-component mixtures of one or more active substances plus a range of co-formulants that serve different purposes, e.g. as stabilizers or preservatives. They are only allowed on the European market if their intended use does not lead to unacceptable risks for the environment. Consequently, the assessment of possible combination effects is a critically important step during the regulatory environmental risk assessment of biocidal products. However, no specific guidance is at hand on how combination effects should be accounted for during the regulatory environmental risk assessment of biocidal products.

Results and Conclusions: A tiered approach was developed that accommodates different data situations, optimizes resource usage, limits biotesting as far as possible and ensures adequate protection of the environment. It mainly builds on using Concentration Addition as a component-based approach for mixture toxicity prediction, complemented by whole product tests where appropriate. Concentration Addition is either approximated by summing up PEC/PNEC ratios or as sums of toxic units. The competing concept of Independent Action was assessed as not being suitable for incorporation into a tiered approach without explicit confirmatory studies, as it might otherwise lead to an underestimation of the actual environmental risk.
\end{abstract}

Keywords: Mixture toxicity, Regulatory environmental risk assessment, Biocides

\section{Background}

Biocidal products are preparations containing one or more active substances, with the intention of "destroying, deterring, rendering harmless, preventing the action of, or otherwise exerting a controlling effect on, any organism by any means other than mere physical or mechanical" (Biocidal Products Regulation, (EU) No 528/2012, BPR) [1]. Biocides are hence closely related to agricultural pesticides and are - due to their high biological activity and potential exposure - of inherent environmental concern. In fact, biocidal products are only allowed to be put on the market of the European Union, if it can be

\footnotetext{
* Correspondence: thomas.backhaus@gu.se

${ }^{1}$ Department of Biological and Environmental Sciences, University of Gothenburg, Carl Skottbergs Gata 22b, 40530 Gothenburg, Sweden Full list of author information is available at the end of the article
}

convincingly demonstrated that no unacceptable risks for the environment result from their intended use.

\section{The European system for biocide authorization}

The authorization of biocides and biocidal products is regulated in the EU according to the rules and procedures laid down in the Biocide Products Directive (BPD, 98/8/EC) [2]. A key precondition for the authorization of a biocidal product is the inclusion of its active substances in the "list of permitted active substances", provided as Annex I or IA of the BPD ("positive list"). Only products that contain active substances that are listed in Annex I/IA are allowed on the EU-market, and the risk from their intended use to man and the environment is then assessed at the national level, complemented by the mutual recognition of authorizations between the EU member states. 
Earlier in 2012 the European Parliament and the EU Council of Ministers adopted the new regulation (EU) No 528/2012 on the authorization of biocidal products (BPR) [1]. It will apply from 1 September 2013 and will replace and repeal the $\mathrm{BPD}$ and will implement a new EU-wide, harmonized system for the authorization for biocidal products. This system will be applied for lowrisk biocidal products and products that have similar use conditions throughout the EU. All other biocidal products are expected to still be subject to authorization by the individual member states. The BPR provisions will also apply to existing active substances being evaluated under the BPD review program.

Biocidal products are usually multi-component mixtures of one or more active substances plus a range of coformulants that serve different purposes, as stabilizers, coloring agents, emulsifiers, solvents, diluents, etc. Additionally, metabolites and degradation products might be formed during and after use of a biocidal product. The overall ecotoxicity of a biocidal product might hence be significantly different from that of each individual ingredient(s) and therefore needs to be assessed during the product authorization phase. In fact, article 19(2) of the BPR states that "the evaluation [...] shall take into account the following factors: [...] cumulative effects, synergistic effects." "Cumulative effects" in the context of the BPR relate to effects from the same compound, emitted from different products, while "synergistic effects" relate to the joint action of different chemicals from one product. This is further elaborated in Annex VI (common principles for the evaluation of biocidal products) which states that the risks associated with the relevant individual components of the biocidal product shall be assessed, taking into account cumulative and synergistic effects.

However, only very limited details on how mixture effects should be considered during the authorization of a biocidal product are provided in the current Technical Note for Guidance on Product Evaluation [3]. In fact, there is currently no agreed guidance available among the European Member States on how to assess the mixture effects from the ingredients of biocidal products, hindering the mutual recognition of authorizations between member states.

The aim of the present text is to bridge this gap and suggest a tiered approach for the adequate consideration of mixture effects during the environmental risk assessment of biocidal products.

\section{Approaches for mixture toxicity assessment}

There are four principal possibilities to assess the toxicity of a mixture of chemicals in general, each with its particular strengths and weaknesses:

1. applying a specific mixture assessment factor (MAF),
2. direct biotesting of the whole product or the environmental mixture resulting from the use of the product,

3. drawing conclusions from available data from similar products (so-called "bridging"), and

4. component-based approaches, such as Concentration Addition (CA).

\section{Mixture assessment factor}

Assessment factors (AF) are commonly used in chemical registration or authorization processes, e.g. in the context of the REACH Regulation (EC) No 1907/2006, the Plant Protection Products Regulation (EC) No 1107/ 2009 , or the BPD. They cover a range of uncertainties, such as laboratory to field extrapolation or acute to chronic extrapolation [4] but are not sized to account for mixture effects. Hence a specific mixture assessment factor (MAF) has been discussed in order to safeguard against unwanted mixture effects from multi-component mixtures of partly unidentified composition [5]. In order to account for this lack of knowledge, the MAF has to incorporate a certain element of conservatism. As the chemical composition of a biocide product that is subject to authorization is known, the MAF is not further considered in the following text.

\section{Direct biotesting}

Testing the whole product is straight forward, does not require any specific methodology and can hence use the same experimental outline as the tests of an individual chemical. However, the BPR states that tests with vertebrate animals can only be conducted as a last resort, i.e. when alternative testing and assessment methods have been exhausted. Furthermore the testing of a biocidal product for its chronic toxicity might be of only limited informative value. The composition of the product might change already during the exposure in biotests, as the different chemicals might have a different stability and distribution between biota, headspace, aqueous media, soil, sediment, etc. Changes in the chemical composition of the initial mixture are most likely even more pronounced if transformation and distribution processes in the environment are taken into consideration.

Such processes can be accounted for by testing the ultimate, environmentally relevant mixture instead of the original product. For example, it might be more relevant to test the leachate of a wood preservative than the original product. The validity of the toxicity data for the assessment then strongly depends on a thorough definition of the underlying exposure scenario. However, there are currently also no agreed guidelines at hand in the EU for the testing of such "environmentally realistic" mixtures (e.g. leachates). 
In principle it would be possible to use data on the toxicity of a product from previous authorizations, assuming that the currently evaluated product is ecotoxicologically similar. However, such strategies have been largely limited to very simple situations as outlined in the bridging principles of the CLP regulation (EC) No $1272 / 2008$. These cover mainly the following situations:

- Batching: It is assumed that the toxicological and ecotoxicologically properties of two batches of a product are identical, as long as its chemical composition does not change substantially.

- Concentration-dependence, dilution: A mixture containing a toxic compound is assumedly less toxic than a similar mixture containing the same compound at higher concentrations. And if a compound is diluted with a less toxic compound, the resulting mixture is assumedly not more toxic than the original compound.

- Substitution: Substitutions involving (eco) toxicologically similar components are assumed to not change the overall toxicity of the mixture.

\section{Component-based approaches}

Component based-approaches are based on the notion that the toxicity of the mixture is a function of the toxicity of the individual compounds. Two simple concepts are established in the literature that systematically link the individual (eco)toxicity of the components of a mixture to its total toxicity, termed Concentration Addition (CA, also known as Dose Addition) and Independent Action (IA, also termed Response Addition), see review by Kortenkamp and colleagues [6]. More elaborated mixture modeling approaches, based on physiologically based pharmakokinetic/pharmakodynamic (PBPK/PD) models [7-9], species-sensitivity distributions (SSDs) $[10,11]$ or dynamic energy budgets (DEBTOX) [12] have to our knowledge not been applied for the environmental risk assessment of biocidal products. This might be due to their substantially higher data demands and because it is currently unclear, whether the increased amount of input data would actually lead to a different regulatory outcome.

A third concept, Effect Summation (ES), is based on the intuitive notion that the effect $E$ of a mixture at a given concentration $c_{M i x}$ simply equals the arithmetic sum of the effects that the $n$ components will provoke if present singly at the concentration at which they are present in the mixture, $c_{i}$ :

$$
E\left(c_{\text {Mix }}\right)=\sum_{i=1}^{n} E\left(c_{i}\right)
$$

However, as it has been repeatedly pointed out, ES lacks a sound pharmacological basis [13-16]. In particular, ES predicts more-than-expected or less-than-expected mixture effects even in the simple case of a so-called "sham combination", a combination of a compound with itself.

$\mathrm{CA}$, on the other hand, adequately describes the joint action of compounds in a sham combination. The concept takes the general mathematical form of:

$$
\frac{c_{\text {mix }}}{E C x_{\text {mix }}}=\sum_{i=1}^{n} \frac{c_{i}}{E C x_{i}}
$$

with $c_{\text {mix }}$ being the total concentration of the mixture and $c_{i}$ denoting the concentrations of each individual compound in the mixture. $E C x_{\text {Mix }}$ and $E C x_{i}$ are the concentrations of the mixture and the $i$-th single substance concentrations, respectively, both provoking an effect of $x \%$.

Alternatively, CA can be formulated as:

$$
E C x_{\text {Mix }}=\left(\sum_{i=1}^{n} \frac{p_{i}}{E C x_{i}}\right)^{-1}
$$

where $p$ denotes the fraction of compound $i$ in the mixture. From a mathematical perspective CA hence simply represents the weighted harmonic mean of the individual $E C x$-values, with the weights just being the fractions $p_{i}$ of the mixture components.

Each concentration $c_{i}$ in equation 2 can also be expressed as a fraction of the corresponding $E C x_{i}$, in which case all fractions in equation 2 take the form of dimensionless so-called "toxic units" (TUs), and the mixture toxicity can be described by the sum of all individual toxic units (STU):

$$
S T U=\sum_{i=1}^{n} T U_{i}
$$

According to CA the individual mixture components behave as if they were dilutions of each other - they only differ in their relative potency and amounts in the mixture. Due to this conceptual idea, CA is thought to describe the joint action of components that have a similar mode or mechanism of action and forms the conceptual basis of the toxicity equivalancy factors (TEF) approaches used for the hazard and risk assessment of e.g. dioxin- or PCB-mixtures $[17,18]$ or the pesticide toxicity index [19].

By activating different molecular effector chains, each component in a mixture of dissimilarly acting chemicals can be thought to contribute to a common biological endpoint, completely independent of the other simultaneously present agents. The resulting combined effect can then be calculated from the effects caused by the individual mixture components by the statistical concept of 
independent random events [20] and the expected combination effect can be mathematically expressed as:

$$
E\left(c_{\text {Mix }}\right)=1-\prod_{i=1}^{n}\left[1-E\left(c_{i}\right)\right]
$$

for the situation that the effect increases with increasing concentrations (e.g. when mortality is the biological endpoint under observation), or

$$
E\left(c_{M i x}\right)=\prod_{i-1}^{n}\left[E\left(c_{i}\right)\right]
$$

when the effect decreases with increasing concentrations (e.g. when survival rates are studied).

The fundamental characteristics and assumptions of CA and IA have been recently summarized and discussed in a series of reviews $[6,10,11,21,22]$. The general notion is that especially CA provides a quite reliable

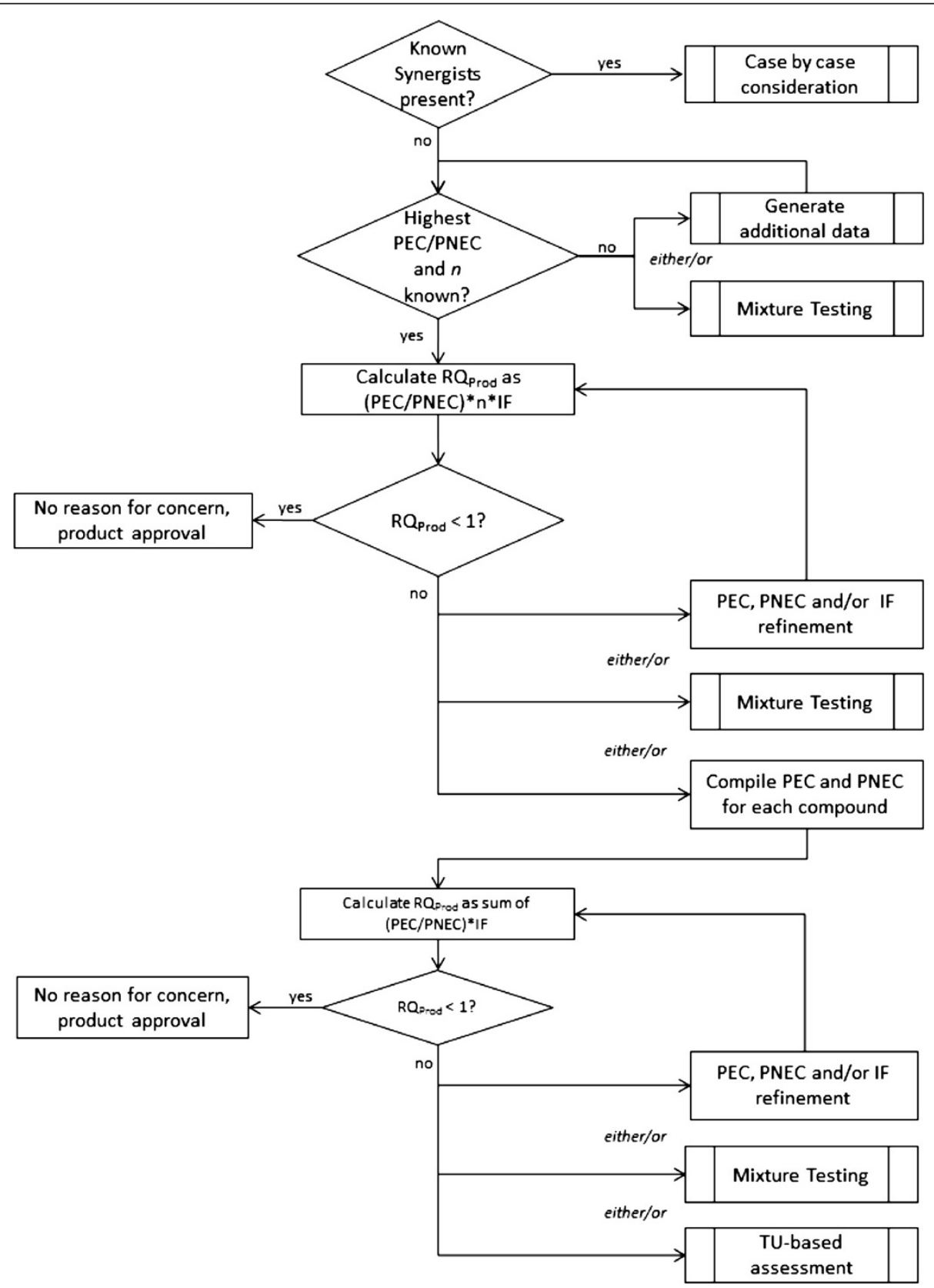

Figure 1 Approach for environmental risk assessment of biocide products. PEC = Predicted Environmental Concentration, PNEC = Predicted No Effect Concentration, RQProd $=$ Risk Quotient for the Product, $\mathrm{TU}=$ Toxic Unit, IF = Interaction Factor, $\mathrm{n}=$ number of compounds in the mixture. 
tool for predicting and assessing the joint ecotoxicity of chemicals, which has been quantitatively analyzed in a range of meta-reviews, often focusing on pesticides and pesticide products $[6,23,24]$.

CA and IA both link the toxicity of individual components (active ingredients, substances of concern, co-formulants) to the overall toxicity of the biocidal product. The major advantage of such methods is their easy adaptability to different exposure situations, e.g. when the chemical composition of a biocidal product changes. They substantially decrease the biotest demands of new biocidal products if they use known active ingredients and/or co-formulants. Similar to the direct testing of a biocidal product for its long-term effects, also the modeling of long-term effects in the environment depends on a sound and complete definition of the underlying exposure scenario.

\section{Results}

\section{Tiered approach for biocide product assessment}

In view of the different options for the environmental risk assessment of a chemical mixture, we suggest the following approach for biocidal product assessment (Figures 1 and 2). It was developed to accommodate various data situations, acknowledging that the initially available data might be quite different for the different products covered by the BPD / BPR.

We base the suggested strategy on component-based approaches as far as possible, as the use of non-testing approaches is already stressed in the BPR in particular with respect to ecotoxicity data for animals. It also facilitates the re-use of existing data for individual ingredients, a factor likely to be increasingly important in the future as the BPR will promote data sharing between applicants. However, the direct testing of a product should be regarded as the "gold standard" for the assessment of acute toxicities or if tests with environmentally realistic mixtures (based on an exposure modeling or monitoring) indicate synergistic interactions.

\section{Discussion}

Any component based approach requires that all "relevant" compounds are included in the assessment, i.e. biologically active chemicals that are present at sufficiently high concentrations. Within the framework of biocide legislation such compounds are termed "substances of concern", i.e. constituents of the biocidal product other than the active ingredient that have "an inherent capacity to cause an adverse effect on humans, animals or the environment" ([1], Art 2e). If no ecotoxicological information is at hand for such ingredients, the only risk assessment option is the direct biotesting of the biocidal product or the resulting environmental mixture, respectively.

A CA-based assessment is a predictive approach and its accuracy is therefore potentially impacted by several confounding factors. These are (i) the stochastic uncertainty of the input data, (ii) the possible amalgamation

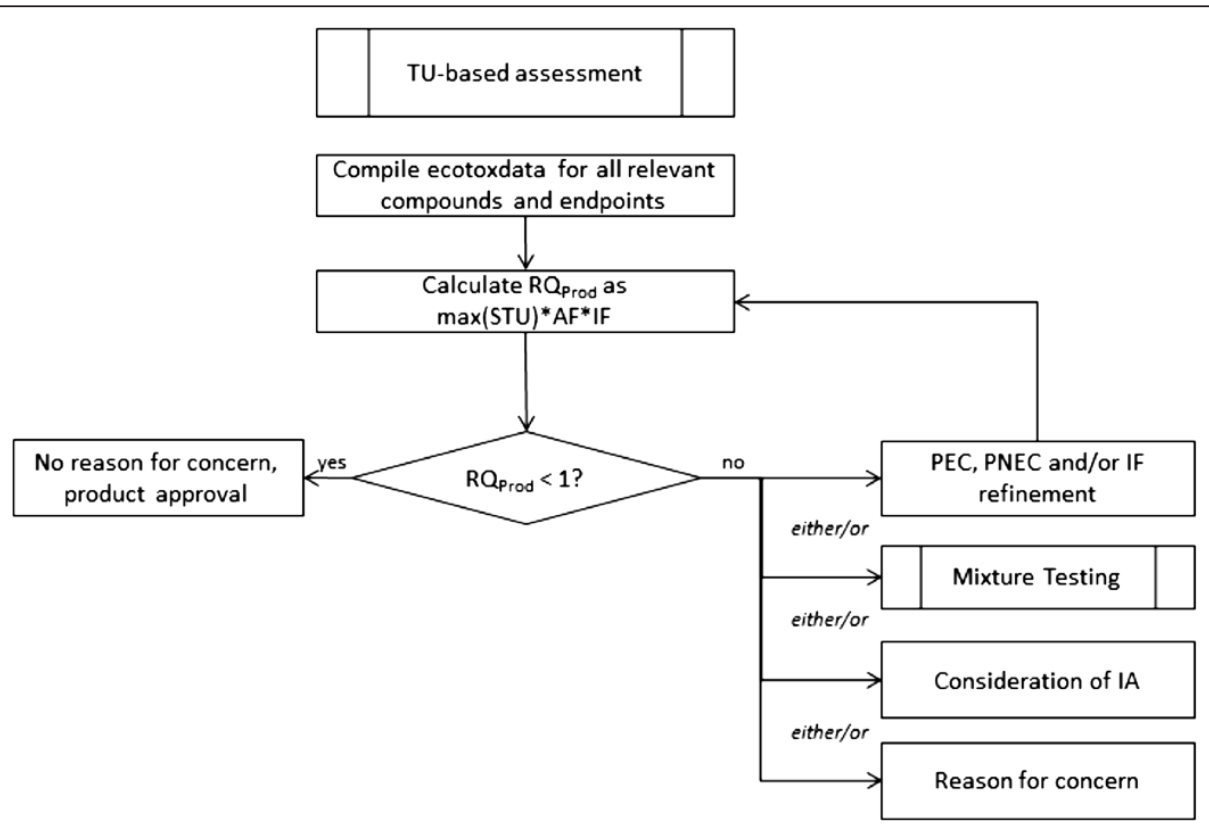

Figure 2 Toxic Unit (TU) based approach for environmental risk assessment of biocide products. PEC = Predicted Environmental Concentration, $\mathrm{PNEC}=$ Predicted No Effect Concentration, $\mathrm{RQ}_{\text {prod }}=$ Risk Quotient for the Product, $\mathrm{TU}=$ Toxic Unit, IF = Interaction Factor, IA = Independent Action, $\mathrm{n}=$ number of compounds in the mixture, $\mathrm{AF}=$ Assessment Factor. 
of single substance data from different species, biotests and endpoints (iii) the non-consideration of relevant ingredients, (iv) the non-consideration of the competing concept of Independent Action, and (v) possible chemical, toxicokinetic and/or -dynamic interactions.

\section{Stochastic uncertainties}

Uncertainties in the input data can stem from e.g. inaccurate measurements or an inherently high variability in the data from a particular bioassay. However, as long as there is no systematic bias in the input data, the stochastic uncertainty of the CA-predicted EC50 is always equal to or smaller than the uncertainty of the most uncertain single substance EC50 value. Hence, if the single substance data are of sufficient quality for the respective single substance risk assessment, no special consideration is needed for a mixture toxicity assessment using CA.

\section{Using data from different bioassays and endpoints}

CA links the toxicity of individual substance to their joint action. The concept implicitly assumes that all single substance toxicity data are recorded for the same species, in the same bioassay and using the same endpoint. In practice, however, the available single substance data might have been recorded under slightly different conditions, using different endpoints or even stem from different species. The following rules should provide guidance for the application of $\mathrm{CA}$ in this context: (i) Toxicity data from different phyla, ecotoxicological endpoints and acute/chronic data should not be combined unless their ecotoxicology implications are similar and adequate assessment factors are considered, see discussion on PEC/PNEC summation below; (ii) if data from more than one species/endpoint are at hand, the most sensitive of the available endpoints/species should be selected for each compound.

\section{Non-consideration of relevant ingredients}

Obviously, if toxic compounds are not considered in a component based assessment, the calculated risk will be an underestimation of the actual risk of the biocidal product, see [25]. It is, however, impossible to provide a general estimate of the magnitude of such an underestimation, as this depends on the concentration and ecotoxicological potency of the compounds that are erroneously not included in the assessment. Therefore, special care has to be taken to ensure that all toxic ingredients are included in a component-based assessment of a biocidal product.

\section{IA in the context of biocide authorization}

The mixtures that make up a biocidal product will usually not be composed of either only strictly similarly or of only strictly dissimilarly acting compounds. Hence, the application of either CA or IA is inherently biased. From the available evidence [6], it is to be expected that the application of CA to a mixture of not entirely similarly acting compounds will lead to a slightly cautious mixture assessment (slight overestimation of risk). Accordingly, using IA for a mixture of at least partly similarly acting substances would often lead to a risk underestimation. Consequently, should IA be used during the authorization of a product, an applicant would need to prove that IA adequately describes the toxicity of the assessed biocidal product. This, however, is only possible by comparing the IA-prediction to experimental data for the product for each considered endpoint. It might therefore be easier and less resource demanding to limit the experimental work to testing the whole product only and to omit a component-based analysis using IA. If IA is considered due to dissimilar modes or mechanisms of action of the ingredients, it should be checked prior to any experimental work whether the actual mixture ratio allows for the possibility that IA might indeed lead to a different regulatory outcome of the assessment, see discussion by Backhaus and Faust, [22]. It should finally be pointed out that only CA can be applied with the typical ecotoxicological data that are documented in the published literature or study summaries, such as EC50 or NOEC-values, see discussion in [6].

\section{Synergistic mixture toxicity}

$\mathrm{CA}$, as well as IA, is based on the assumption that the compounds in a mixture do not interact, neither chemically nor in their toxicokinetic and-dynamic phases. Such interactions might cause synergisms, i.e. a mixture toxicity that is higher than expected by both concepts. Although comparatively rare in general [6], several examples of synergistic interactions can be found in the literature. They are mainly restricted to mixtures with a few (usually two) compounds, which is exactly the situation that is relevant within the context of biocidal product authorization. For example, the combination of zinc-pyrithione and copper, two antifouling biocides, shows a clearly higher toxicity than predicted by CA in a range of bioassays, due to the trans-chelation of zincpyrithione to the significantly more toxic copperpyrithione [26]. Mixtures of organophosphates and carbamates (insecticides) were consistently more toxic to fish than predicted by $\mathrm{CA}$, despite their similar mechanisms of action [27]. This is most likely caused by the inhibition of organophosphate biotransformation to their inactive dicarboxylic acid derivates by carbamates.

We therefore suggest to initially penalize CA-based assessments with an additional assessment factor, termed "IF" (Interaction Factor), in particular if no ecotoxicity 
data for the product in question are at hand. This factor shall account for the possibility of synergistic interactions (higher mixture toxicity than predicted due to chemical, toxicokinetic and/or -dynamic interactions). It should be emphasized that the IF is not meant to account for any of the other potential error sources that were outlined above.

A review on the predictive power of CA for pesticide mixtures concluded that in less than $5 \%$ of the published studies the experimental toxicity exceeded the predictions by a factor of 2 or more [23]. A recent reanalysis of data available to the German Federal Environment Agency evaluated the predictive power of CA for commercial pesticide products and came to the conclusion that in $50 \%$ of the cases CA predicted the experimental toxicity correctly within a factor of 2 , while in $50 \%$ of the investigated products significant deviations of up to a factor of 800 in one situation were observed [24]. These studies do not allow estimating an IF, as it is unclear to which extent each of the factors listed above (stochaistic uncertainty, interactions, incomplete consideration of all components present) was responsible for the overall deviations between CA-predictions and observations. But as the general chance of underestimating the risk by more than a factor of 2 seems to be low for the majority of cases, an IF of 2 currently seems sufficiently protective. However, it should be pointed out, that empirical evidence on the joint effects of coformulants and active ingredients of biocidal products is scarce, and more empirical evidence is urgently needed. Consequently, if available evidence is at hand, the IF might have to be set to a value greater than 2 , or the IF could be decreased down to 1 for a specific product if sufficient evidence is provided (see Figure 1).

Inert compounds such as water or non-soluble pigments are chemicals that do not show any toxic effects, even at excessive concentrations and do not interact with other chemicals present. Hence they do not have an impact on the mixture toxicity assessment, as both concepts assume that they do not contribute to the overall toxicity of the product.

Inert compounds need to be clearly differentiated from compounds that are not inherently toxic per se, but still are biologically active. Piperonyl butoxide for example would fall into this group, as the compound itself is not biocidal, but increases the toxicity of other biocides by inhibiting their cytochrome P450-driven metabolization. Such known synergists might lead to serious toxicity and risk underestimations, and hence have to be considered specifically in a case-by-case manner, in amendment to the proposed scheme.

\section{Requested input data}

The outlined strategy keeps the initial data demands as low as possible (i.e. optimizes resource efficacy and limits unnecessary testing), while at the same time ensuring an adequate protection of the environment according to the philosophy and approaches of the BPD/ BPR. The minimum requested set of data for a component-based assessment (Figure 1) consists of (i) solid and complete information on the product composition, and (ii) the PEC/PNEC ratio for the most risky compound, typically the active ingredient. This implies that the PEC/PNEC ratio of all other compounds is known to be lower, which should be adequately demonstrated. As only semi-quantitative data are needed for this purpose, QSAR-estimates, hazard classification data from classification and labeling according to the CLP Regulation (EC) No 1272/2008, censored toxicity data (e.g. from limit tests) and simple exposure estimates should be sufficient.

The final risk of the product is then estimated as

$$
R Q_{\text {Product }}=n \times I F \times\left(\frac{P E C}{P N E C}\right)_{\max }
$$

where $n$ is the number of relevant compounds in the mixture, while the interaction factor IF accounts for the possible occurrence of toxicokinetic or -dynamic interactions. Eq. 7 is providing a first tier worst-case risk estimation, assuming that all compound have a risk quotient equal to (PEC/PNEC) $\max$.

This first tier allows an initial precautionary assessment already with a very limited set of data. If there are no reasons for concern (i.e. $R Q_{\text {Product }}<1$ ), no further testing or data evaluation is required.

Four options exist, if there are potential reasons for concern ( $\mathrm{RQ}_{\text {Product }}>1$, Figure 1): (i) the $\mathrm{PEC}$ and/or PNEC estimate of the most risky compound might be refined by providing additional ecotoxicological data and/or exposure estimates. Such an effort might be worthwhile in particular if the PNEC assessment is based only on the so-called base-set of data, i.e. short-term toxicity data for algae, daphnids and fish, according to ECHA (2008); (ii) evidence from e.g. dedicated experimental mixture studies with these or other representatives of the compounds / compound classes in question are provided, in order to allows a better estimation of the IF; (iii) the whole mixture (biocidal product) might be subjected to direct biotesting, considering the limitations as outlined above; or (iv) a more detailed component-based assessment is carried out that uses quantitative risk estimates not only for the most risky compound, but for every relevant compound.

If the PEC/PNEC ratios are at hand for all relevant ingredients, the risk quotient of the product $R Q_{\text {Prod }}$ can be simply estimated by the PEC/PNEC sum: 


$$
R Q_{\mathrm{Prod}}=R Q_{\mathrm{PEC} / \mathrm{PNEC}}=\sum_{i=1}^{n}\left(\frac{P E C}{P N E C}\right)_{i} \times I F
$$

Summing up PEC/PNECs is mentioned in the Technical Notes for Guidance as one option for biocide product assessment (ECB, 2008). However, it should be pointed out that eq. 8 is fundamentally different from CA (eq. 4), as the PNECs from the various compounds might be based on data from completely different endpoints and species. Hence eq. 8 violates one of the fundamental assumptions of CA. Consequently, the use of PEC/PNEC sums has recently been advised against in the preliminary opinion on mixture toxicity assessment as put forward by the EU scientific committees [28]. However, it can be proven that eq. 8 provides a conservative approximation of $\mathrm{CA}$ [22]. Furthermore, it is a major advantage of the PEC/PNEC sum (eq. 8) that it can be applied even if different amounts of data are available for the different compounds in the product, for example when an extended data set including chronic ecotoxicity data is at hand for the active ingredient, but only base-set data are available for the other substances of concern. For a more detailed discussion on the use of PEC/PNEC sums see [22].

Should eq. 8 still indicate reason for concern $\left(\mathrm{RQ}_{\text {Product }}>1\right)$, the following options exist: (i) direct product testing (but see discussion above); (ii) a refinement of the PEC- and/or PNEC-values by providing additional information on the exposure and / or hazard characterization of the compounds, especially those that dominate the sum of PEC/PNECs, or (iii) the application of CA in the form of a sum of toxic units (STU) as follows (Figure 2):

$$
\begin{aligned}
R Q_{\text {Prod }}= & R Q_{S T U} \\
= & \max \left(S T U_{\text {endpoint } 1}, S T U_{\text {endpoint } 2}, \ldots, S T U_{\text {endpoint } m}\right) \\
& \times A F \times I F \\
= & \max \left(\sum_{i=1}^{n} \frac{P E C_{i}}{E C 50_{i, j}}, \sum_{i=1}^{n} \frac{P E C_{i}}{E C 50_{i, j}}, \ldots, \sum_{i=1}^{n} \frac{P E C_{i}}{E C 50_{i, m}}\right) \\
& \times A F \times I F
\end{aligned}
$$

That is, the sum of toxic units (STU) is calculated for each ecotoxicological endpoint, which is species-specific. $A F$ denotes the resulting assessment factor, in accordance with [4].

The maximum STU indicates which endpoint for which species is expected to be most sensitive to the biocidal product in question and is hence used for the final assessment. It can be mathematically proven that the risk quotient that results from summing up PEC/ PNECs (eq. 8) is always equal or higher than the maximum STU according to eq. 9 [22]. Their precise relationship depends on the ecotoxicological profiles of the compounds in the mixture. In case of dissimilar profiles, the ratio between the application of eq. 8 and 9 approaches the theoretical maximum of $m$, which is number of considered endpoints. If the compounds have almost the same ecotoxicological profiles, which can be expected e.g. for a mixture of simple organic solvents, then the risk quotients from both equations become identical.

The maximum ratio between $\mathrm{RQ}_{\mathrm{PEC} / \mathrm{PNEC}}$ and $\mathrm{RQ}_{\mathrm{STU}}$ of $m$ provides a convenient decision criterion on whether the detailed data collection or production in order to conduct a refined assessment based on RQSTU (eq. 9) might influence the regulatory outcome: if $\mathrm{RQ}_{\mathrm{PEC} / \mathrm{PNEC}}$ is higher than $m, \mathrm{RQ}_{\mathrm{STU}}$ will always be above 1, i.e. indicate reason for concern.

Employing eq. 9 requires that data for all relevant compounds are available for all endpoints, as it would otherwise be impossible to determine the maximum of all organism- and endpoint-specific STUs and an appropriate overall assessment factor (AF). This makes an application of equation 9 - although it most closely follows the conceptual idea of CA - rather demanding.

A risk quotient exceeding one might be caused by the overestimation that results from the application of CA to a mixture of not entirely similarly acting compounds. Details on how to estimate this possible overestimation are provided by Junghans and colleagues [29], Backhaus and colleagues [22] and Altenburger et al. [30]. The direct testing of the biocidal product might provide additional insight, given that a substantial risk overestimation by $\mathrm{CA}$ is possible, which depends on the number of involved compounds, their toxicity and ratio in the mixture. Otherwise there would be a clear indication for a reason for environmental concern, which would call for appropriate risk management strategies.

\section{Conclusion}

The component based assessment of biocide products is a robust approach to account for mixture toxicity when incorporated into an appropriate tiered scheme. Particularly, it allows to focus attention and efforts on those cases for which mixture effects are of potential concern, and it initially uses only the available toxicity information of the individual components for this purpose. The presented tiered approach might hence serve as a template for the development of specific guideline documents in support of the new biocide regulation (EU) $528 / 2012$. In view of the novelty of the regulation details of the presented approach might require fine-tuning, as soon as more practical experience has been collected. In particular the use and initial size of the IF might warrant later review and perhaps adjustment. 


\section{Competing interests}

Thomas Backhaus, Rolf Altenburger and Michael Faust declare no competing interests, neither financial nor otherwise. Anja Kehrer, Daniel Frein, Tobias Frische, Per Johansson and Tobias Porsbring are actively involved in the regulatory risk assessment of biocides on a member state or European level.

\section{Authors' contributions}

All authors participated in the development of the outlined approach during a series of meetings and workshops that were conducted under the auspice of the German Federal Environment Agency in the context of the project "Ecotoxicological combined effects from chemical mixtures" (FKZ 370965404). TB drafted the manuscript. All authors read and agreed on the final manuscript.

\section{Disclaimer}

This paper presents the opinion of its authors and hence does not necessarily reflect the opinion or the policies of the German Federal Environment Agency, the Swedish Chemicals Inspectorate or the European Commission

\section{Acknowledgements}

Funding by the German Federal Environment Agency (FKZ 370965404) is gratefully acknowledged.

\section{Author details}

${ }^{1}$ Department of Biological and Environmental Sciences, University of Gothenburg, Carl Skottbergs Gata 22b, 40530 Gothenburg, Sweden. 2UFZ - Helmholtz Centre for Environmental Research, UFZ, Department Bioanalytical Ecotoxicology, Permoser Str. 15, 04318 Leipzig, Germany. ${ }^{3}$ Faust \& Backhaus, Environmental Consulting, Bremen, Germany. ${ }^{4}$ Federal Environment Agency, 06844 Dessau-Roßlau, Germany. ${ }^{5}$ Federal Environment Agency, 06844 Dessau-Roßlau, Germany. 'Swedish Chemicals Agency, 17213 Sundbyberg, Sweden. ${ }^{7}$ Federal Environment Agency, 06844 Dessau-Roßlau, Germany. ${ }^{8}$ Institute for Health and Consumer Protection, European Commission's Joint Research Centre, 21027 Ispra, Italy.

\section{Received: 16 October 2012 Accepted: 29 January 2013}

Published: 28 February 2013

\section{References}

1. Regulation (EU) No 528/2012 of the European Parliament and of the Council of 22: concerning the making available on the market and use of biocidal products. Official Journal L 2012, 167:1-123.

2. Directive $98 / 8 /$ EC of the European Parliament and of the Council of 16 : concerning the placing of biocidal products on the market. Official Journal L 1998, 123:1-0063.

3. European Chemicals Bureau: Technical Notes for Guidance in support of Annex VI of Directive 98/8/EC of the European Parliament and the Council concerning the placing of biocidal products on the market; 2008.

4. European Chemicals Agency (ECHA): Characterisation of dose [concentration]-response for environment. In Guidance on information requirements and chemical safety assessment; 2008.

5. Backhaus T, Faust M, Blanck H: Hazard and Risk Assessment of Chemical Mixtures under REACH-State of the Art, Gaps and Options for Improvement Swedish Chemicals Agency Report; 2010. PM 3/10. 2010. www.kemi.se/ Documents/Publikationer/Trycksaker/PM/PM3_10.pdf.

6. Kortenkamp A, Backhaus T, Faust M: State of the Art Report on Mixture Toxicity. Directorate General for the Environment: Report to the EU Commission; 2009. ec.europa.eu/environment/chemicals/pdf/report_Mixture\%20toxicity.pdf.

7. Lowit A, Darit C, Powers F, Blancato J, Setzer RW, Conolly R: Physiologically-based pharmacokinetic/pharmacodynamic modeling and cumulative risk assessment. EPA Science Forum: Case study for the N-methyl carbamate pesticides; 2004, Washington, DC, June 1-3, 2004.

8. Teuschler LK, Rice GE, Wilkes CR, Lipscomb JC, Power FW: Feasibility study of cumulative risk assessment methods for drinking water disinfection by-product mixtures. J Tox Env Health A 2004, 67(8-10):755-77.

9. Conolly R, Wang J, Tan Y: Physiologically based pharmacokinetic (PBPK) modeling as an alternative to relative potency factors (RPFs) in cumulative risk assessment: An example with acetylcholinesterase(AChE) inhibiting pesticides. Toxicological Science 2005, 84(S-1):34
10. de Zwart D, Posthuma L: Complex mixture toxicity for single and multiple species: proposed methodologies. Env Tox Chem 2005, 24(10):2665-2676.

11. Posthuma L, Richards S, de Zwart D, Dyer S, Sibley P, Hickey C, Altenburger R: Mixture extrapolation approaches. In Extrapolation practice for ecotoxicological effect characterization of chemicals. Edited by Solomon K, Brock T, de Zwart D, Dyer S, Posthuma L, Richards S, Sanderson H, Sibley P, van den Brink P. Boca Raton (FL): Taylor \& Francis; 2008:137-185.

12. Baas J, Jager T, Kooijman B: A review of DEB theory in assessing toxic effects of mixtures. Sci. Tot. Env. 2010, 408(18):3740-3745.

13. Greco WR, Bravo G, Parsons JC: The search for synergy: a critical review from a response surface perspective. Pharmacol Review 1995, 47(2):331-85.

14. Berenbaum MC: The expected effect of a combination of agents: the general solution. J Theor Biol 1985, 114:413-31.

15. Boedeker W, Altenburger R, Faust M, Grimme LH: Synopsis of concepts and models for the quantitative analysis of combination effects: from biometrics to ecotoxicology. Arch Compl Env Studies 1992, 4(3):45-53.

16. Boedeker W, Drescher K, Altenburger R, Faust M, Grimme LH: Combined effects of toxicants: the need and soundness of assessment approaches in ecotoxicology. Sci Total Environ 1993, 134(Supp2):931-939.

17. van den Berg M, Birnbaum L, Bosveld AT, Brunstrom B, Cook P, Feeley M, Giesy JP, Hanberg A, Hasegawa R, Kennedy SW, Kubiak T, Larsen JC, van Leeuwen FX, Liem AK, Nolt C, Peterson RE, Poellinger L, Safe S, Schrenk D, Tillitt D, Tysklind M, Younes M, Waern F, Zacharewski T: Toxic equivalency factors (TEFs) for PCBs, PCDDs, PCDFs for humans and wildlife. Environ Health Perspect 1998, 106(12):775-792.

18. US Environmental Protection Agency: Framework for application of the toxicity equivalence methodology for polychlorinated dioxins, furans, and biphenyls in ecological risk assessment; 2008. EPA 100/R-08/004.

19. Munn MD, Gilliom RJ, Moran PW, Nowell LH: Pesticide Toxicity Index. US Geological Survey: US Department of the Interior; 2006. $2^{\text {nd }}$ edition.

20. Bliss Cl: The toxicity of poisons applied jointly. Ann J Appl Biol 1939, 26:585-615.

21. Altenburger R, Greco W: Extrapolation concepts for dealing with multiple contamination in environmental risk assessment. Int Env Ass Man 2009, 5:62-68.

22. Backhaus T, Faust M: Predictive environmental risk assessment of chemical mixtures: a conceptual framework. Env Sci Techn 2012, 46(5):2564-2573.

23. Belden JB, Gilliom RJ, Lydy MJ: How well can we predict the toxicity of pesticide mixtures to aquatic life? Int Env Ass Man 2007, 3:364-372

24. Coors A, Frische T: Predicting the aquatic toxicity of commercial pesticide mixtures. Env Sci Europe 2011, 23(22).

25. Coors A, Dobrick J, Möder M, Kehrer A: Mixture toxicity of wood preservative products in the fish embryo toxicity test. Env Tox Chem 2012, 31(6):1239-1248

26. Bao WWW, Leung KMY, Kwok KWH, Zhang AQ, Lui GCS: Synergistic toxic effects of zinc pyrithione and copper to three marine species: Implications on setting appropriate water quality criteria. Marine Poll Bulletin 2008, 57:616-623

27. Laetz CA, Baldwin DH, Collier TK, Hebert V, Stark JD: NL: The synergistic toxicity of pesticide mixtures: Implications for risk assessment and the conservation of endangered Pacific salmon. Environ Health Perspect 2009, 117(3):348-353.

28. Scientific Committee on Consumer Safety (SCCS) \& Scientific Committee on Health and Environmental Risks (SCHER) \& Scientific Committee on Emerging and Newly Identified Health Risks (SCENIHR): Toxicity and Assessment of Chemical Mixtures. Preliminary Opinion approved for Public Consultation; 2011.

29. Junghans M, Backhaus T, Faust M, Scholze M, Grimme LH: Application and validation of approaches for the predictive hazard assessment of realistic pesticide mixtures. Aquat Toxicol 2006, 76(2):93-110.

30. Altenburger $\mathrm{R}$, Walter $\mathrm{H}$, Grote M: What contributes to the combined effect of a complex mixture? Environ Sci Technol 2004, 38:6353-6362.

\section{doi:10.1186/2190-4715-25-4}

Cite this article as: Backhaus et al:: Proposal for environmental mixture risk assessment in the context of the biocidal product authorization in the EU. Environmental Sciences Europe 2013 25:4. 